\title{
Qualität und Qualitäts- messung in der Pflege aus ethischer Perspektive
}

\author{
Christiane Luderer und Gabriele Meyer \\ ○ Der/die Autor(en) 2018 \\ K. Jacobs et al. (Hrsg.), Pflege-Report 2018 \\ https://doi.org/10.1007/978-3-662-56822-4_2
}

\section{Zusammenfassung}

Aus ethischer Perspektive ist eine ganzheitliche, auf die Sichtweise der Patientinnen und Patienten ausgerichtete Erfassung von Pflegequalität anzustreben. "Unsichtbare Pflegetätigkeiten können dann mehr Berücksichtigung erfahren. Ethisch relevante Indikatoren der Pflegequalität (z. B. Moralvorstellungen, Haltungen, Affektkomposition, Beziehungsfähigkeit) sind schwer zu messen und nur für einige sind bislang Messinstrumente verfügbar. Neben der Messbarkeit ist es wichtig, die Reflexivität der Pflegenden dahingehend zu stärken. Regelwerke wie der Ethikkodex können Pflegende dabei unterstützen, diese Werte auch bei hoher Arbeitsdichte zu reflektieren und Arbeitsaufgaben zu priorisieren.

From an ethical point of view, the assessment of nursing quality should aim to reflect a holistic, patient-oriented perspective. Hence, credit might be given to so far «invisible" nursing activities. Ethically relevant indicators of nursing quality (e.g. morality, attitudes, affect composition, relationship skills) are difficult to measure and only few instruments for selected indicators are available. Beyond measurement, nurses' ethical reflexivity remains to be strengthened. The Code of Ethics might support nurses to reflect these values even if the workload is high and enable them to prioritize working tasks.

\subsection{Ausgangssituation: das Ver- ständnis von Pflegequalität}

Was Pflegequalität bedeutet und wie sie zu erfassen ist, hat sich im Laufe der letzten 50 Jahre deutlich verändert. Von einer ursprünglich in simplen Kategorien auf einer Skala gut bis ausreichend definierten Pflegequalität reichte die Entwicklung über Ansätze, die Zielerreichungsgrade fokussieren, zu einem Konzept, das die Komplexität der Triade Patient/Patientin - Pflege - System einschließlich der An- und Zugehörigen berücksichtigen möchte (Hasseler et al. 2016). Die Effekte der pflegerischen Qualität werden zunehmend indikatorgestützt durch das Konzept der evidenzbasierten Pflege aufgezeigt oder auch infrage gestellt, sodass Pflegende sich auf aktualisierbare Empfehlungen und damit lebenslanges Lernen einlassen müssen (Büscher und Blumenberg 2018). Die wachsende Vernetzung mit weiteren Qualitätsdimensionen, wie dem interprofessionellen Arbeiten (Frenk et al. 2010) oder der Berücksichtigung interkultureller und interreligiöser Bedürfnisse (Leufgen 2018), führt zu einer Verquickung verschiedener Determinanten von Pflegequalität, die eben nicht auf die Einhaltung pflegerischer Standards reduzierbar ist. Dies legitimiert, die ethische Fundierung der derzeit geltenden Qualitätsbegriffe in der Pflege zu reflektieren. 
Eine qualitativ hochwertige Pflege zeichnet sich dadurch aus, dass sie an den Bedürfnissen und Präferenzen der Pflegeempfängerinnen und -empfänger ausgerichtet ist und Maßnahmen mit nachgewiesener Wirksamkeit unter Berücksichtigung der Wirtschaftlichkeit anbietet (Hasseler et al. 2016).

In dieser Verschmelzung verschiedener Definitionen wird bereits die ethische Komponente von Pflegequalität sichtbar: Was ist, wenn sich Bedürfnisse und Bedarf aus den verschiedenen Blickwinkeln unterschiedlich gestalten? Was ist, wenn individuelle Präferenzen den verfügbaren Ressourcen zuwiderlaufen? Wie gehen Pflegende damit um, wenn für nachgefragte pflegerische Maßnahmen der wissenschaftliche Nachweis aussteht oder wenn beim Abwägen der Werte und der Allokation der verfügbaren Ressourcen das Gewissen plagt?

\subsection{Der Orientierungsrahmen Pflegender als Säule der Pflege- qualität}

Das derzeit geltende Verständnis von Pflegequalität rekurriert auf die Abbildung der Qualitätsparameter in der Performance selbst oder in repräsentierenden Outcomes, die als Indikatoren oder Kriterien bezeichnet werden (Hasseler et al. 2016). Die jeweilige Performance einer pflegerischen Situation wird jedoch auch durch verschiedene Determinanten außerhalb der methodischen, sozialen und fachlich-kognitiven Kompetenzen konstituiert.

Einerseits sind hier affektive bzw. affektbeeinflussende Persönlichkeitsmerkmale zu benennen, die von den Pflegenden selbst nur bedingt reflektiert werden können und die auf deren Handeln wirken. Die Affektlogik (Ciompi 2016; Damasio 2015) ist nicht nur aus lerntheoretischer Perspektive interessant (ein moderates Erregungsniveau ist hilfreich für nachhaltiges Lernen), sondern sie wirkt ebenso nachhaltig auf die Gestaltung von Interaktionsprozessen. Dies wird jedoch in nur geringen Anteilen in den Curricula der Gesundheitsfachberufe berücksichtigt, sodass eine bewusste Affektsteuerung (z. B. durch gezielte Achtsamkeitstrainings) nur einer kleinen Gruppe der Pflegenden zugänglich ist (Irving et al. 2009).
Weiterhin wirken in der jeweiligen Performance die Einstellungen der Pflegenden, die als relativ stabile Vorbedingung des Handelns gelten (Ajzen 2011). Eine Einstellung erhöht die Wahrscheinlichkeit einer zu erwartenden Handlung einer Person in bestimmten Kontexten, z. B. in Situationen, in denen durch unterschiedliche Perspektiven und Bedürfnisse Konfliktpotenzial besteht.

Gekoppelt an die Einstellungen und situativ konstituierten Affektkompositionen wirken auch die Moralvorstellungen der Pflegenden auf deren Handeln (Jormsri et al. 2005). Daran geknüpft ist das moralisch bedingte Verpflichtungsgefühl, das eine Erklärung von Zuständigkeiten und Engagement bewirkt (Amiri et al. 2018; Fida et al. 2016).

Die affektive Natur, (latente) Einstellungen und das Moralverständnis einer Person gelten als schwer messbare Konstrukte, die in der Überprüfung der Pflegequalität bislang kaum eine Rolle spielen (Kuis et al. 2014). Doch genau jene Konstrukte sind es, die bestimmen,

- ob Pflege außerhalb von qualitätsüberprüfenden Situationen nachhaltig entsprechend den vorgegebenen Kriterien »qualitätskonform « umgesetzt wird, z. B. in der Einhaltung hygienischer Richtlinien oder in der Wahrung der Persönlichkeitsrechte Pflegebedürftiger;

- ob Zuständigkeiten erkannt und wahrgenommen werden in den Kontexten

- der anwaltschaftlichen Vertretung von Patientinnen und Patienten und deren An- und Zugehörigen in hierarchisch geprägten Interaktionen im Gesundheitswesen,

- der intra-und interprofessionellen Zusammenarbeit,

- der Wahrung interkultureller und interreligiöser Bedürfnisse,

- der Identifikation besonderer Patientenbedürfnisse, z. B. in Fragen der Palliation, - der beruflichen Interessenvertretung;

- ob reflexiv mit Situationen umgegangen wird und wie Fehlern begegnet wird;

- ob und in welchem Maße eine Bereitschaft zur Veränderung und zu Investitionen besteht und entsprechendes Engagement übernommen wird. 
Diese Vorbedingungen pflegerischen Handelns als intrapsychische Konstellationen gestalten eine objektive Erfassung mit wenig Aufwand schwierig. Die grundlegenden Herausforderungen der Einstellungsforschung (Ajzen 2011), das Phänomen der sozialen Erwünschtheit (Kowalski et al. 2018) und die unterschiedliche Steuerbarkeit der Affektkontrolle (Ciompi 2016), erlauben keine objektiven Rückschlüsse oder Vorhersagen auf die qualitativen Merkmale des pflegerischen Handelns einer Person. Paradoxerweise werden Haltungen oder der innere Orientierungsrahmen des Handelns oft erst dann reflektiert, wenn die Pflegequalität durch Konflikte oder Spannungen infrage gestellt wird (Staudhammer 2018).

Die Frage nach der ethischen Perspektive von Qualität und Qualitätsmessung impliziert aus der Perspektive der Ethik die Reflexion der Wertesysteme; der inneren, oft nur latent bewussten Orientierungsmuster, die von Pflegenden selbst als bedeutsame Kompetenzen beschrieben werden (Zhang et al. 2001). Ist das, was in den Ausbildungen im Themenbereich zum beruflichen Selbstverständnis in der Pflege gelehrt wird, auch das, was den Bezugsrahmen der Werte der zukünftig Pflegenden ausmacht (Schwarz 2009)? Inwieweit werden der innere Orientierungsrahmen und das Wertesystem von Pflegenden im Rahmen von Einstellungsverfahren oder der Qualitätsentwicklung und Qualitätsmessung berücksichtigt? Wie lassen sich diese dem Handeln zugrunde liegenden Konstrukte beurteilen oder gar als Qualitätskriterium definieren? Und welche Konsequenz hat dies für den beruflichen Werdegang einer Pflegefachkraft? Der derzeitige Forschungsstand bleibt Antworten auf diese Fragen schuldig (Wilson et al. 2015).

\subsection{Erhalt der Würde als Qualitäts- merkmal?}

Das Moralverständnis und die Einstellungen von Pflegenden werden von Pflegeempfängerinnen und Pflegeempfängern intensiver als die messbaren Qualitätsmerkmale der Pflege reflektiert, da insbesondere die Soft Skills dadurch mitbestimmt werden. Das Maß an Freundlichkeit, Sympathie, Empathie und Komfort wird von den Empfänger/-innen von Pflege weitaus differenzierter erfasst (Sinclair et al. 2017) als die korrekte Qualität in der Umsetzung pflegerischer Interventionen. »Korrektheit» wird vor allem über den möglichst hohen Komfort bzw. die Schmerzarmut einer Intervention und die wahrgenommene Teamarbeit definiert (Attree 2001; Beattie et al. 2014; Gillespie et al. 2017).

Der ethische Aspekt von Qualität aus der Sicht der Empfänger/-innen stellt sich besonders in Situationen heraus, in denen Pflege als unzureichend oder unpassend beschrieben wird: Hier ist es vor allem die als Patientenrecht geltende Achtung der Würde (WHO 1994), die von Pflegeempfängerinnen und -empfängern und deren Vertreter/-innen vermisst wird (Chochinov 2013). Würde impliziert Wertschätzung und Gerechtigkeit, Mitbestimmung und Komfort in körperlichen, psychischen und spirituellen Bedürfnissen. Dem weit auslegbaren Konstrukt (Jacobson 2007) liegt einerseits eine Basiserwartung, ein gesellschaftliches Grundverständnis und andererseits ein Recht und ein nicht von außen reduzierbares, ein »unverletzliches « Gut zugrunde (Bielefeldt 2004, S. 23f.). Würde gilt als fundamental, sie kann einem Menschen nicht genommen werden (ebda.). Sie als Qualitätsmerkmal zu definieren, stellt die Selbstverständlichkeit der Achtung von Würde im pflegerischen Alltag infrage und räumt deren Missachtung ein. Im Kontext des Umgangs von Menschen miteinander wird Würde als die einzigartige Bestimmung des Seins einer Person definiert, eine Anerkennung der Einzigartigkeit, ein »Sehen« des Menschen (Chochinov 2013). Unter diesem Paradigma wird die Qualität der Pflege aus ethischer Perspektive unter den derzeitigen Bedingungen des Arbeitens im Krankenhaus, in der ambulanten Pflege oder in Einrichtungen der Seniorenpflege vor allem von Pflegenden selbst kritisch betrachtet (Chochinov 2013; Heijkenskjöld et al. 2010; Stievano et al. 2013). Der Zusammenhang zwischen pflegesensitiven Outcomes und Pflegeverhältniszahlen ist vielfach hergestellt (Aiken et al. 2014; Schreyögg und Milstein 2016). Bei der aktuell als hoch empfundenen Arbeitsdichte durch steigende Zahlen von Pflegebedürftigen und komplexen Anforderungen (Isfort 2018) sehen die Pflegenden den Anspruch an die nötige emotionale Zuwendung und emphatische Pflege nicht als erfüllt, zumal viele der von ihnen geleisteten unsichtbaren 
Tätigkeiten nicht wertgeschätzt werden (Sasso et al. 2017). Amiri et al. (2016) verdeutlichen dabei einen Zusammenhang von moralischen Konflikten Pflegender und schlechter Pflegequalität, was wiederum zu einer Verschärfung der Arbeitssituation führen kann (Schürmann und Gather 2018).

\subsection{Das Problem der Messbarkeit: Würde als pflegesensitiver Indikator}

Um diese ethische Perspektive konsequent zu verfolgen, wäre es nötig, dass die Qualitätsmessung in der Pflege nicht allein auf die korrekte Auswahl und Umsetzung von Pflegemethoden, Techniken und Konzepten fokussiert, sondern auch die Einbettung dieser Angebote in das konkrete pflegerische Szenario einschließlich der »unsichtbaren Pflegetätigkeiten« (Sasso et al. 2017) überprüft. Hierzu zählen beispielsweise der Vertrauensaufbau (Dinç u. Gastmans 2013), die zurückhaltende Beobachtung oder psychologische Zuwendung zu den Patientinnen und Patienten (Sasso et al. 2017). Ein Ansatz dafür ist die Berücksichtigung der pflegerischen Tätigkeiten, die bislang unter dem »Missed-Care«-Konzept erfasst werden (ebda.).

Eine einwandfreie Technik von pflegerischen Angeboten allein sichert noch nicht die Pflegequalität, wenn sie lediglich in Überprüfungssituationen, im falschen Rahmen oder kommunikativ unachtsam bzw. entwertend umgesetzt und nicht bewusst in alltägliche Handlungsroutinen integriert wird (Godin et al. 2008). Um die Angemessenheit von Zeit und Rahmenbedingungen in Synchronisation mit den Bedürfnissen der Pflegebedürftigen zu erfassen, benötigen Pflegende ein ausgeprägtes Maß an Perspektivenübernahmefähigkeit, in der Literatur als Feinfühligkeit oder Empathie bezeichnet (Batson 2009; Bischoff-Wanner 2002). Gerade hier offenbart sich jedoch das Problem der Messbarkeit dieser Säule von Pflegequalität, denn die bestehenden Instrumente zur Selbsterfassung oder Fremderfassung von Empathie sind in ihrer Aussagefähigkeit begrenzt und zum Teil aufwändig (Yu und Kirk 2009). Beurteilungen der empathischen Kompetenzen finden vor allem im Rahmen der praktischen Ausbildung statt und werden von den Lernenden gut angenommen (Brunero et al. 2010; Foster et al. 2015; Larocco 2010; Walker und Mann 2016). Für die beruflich Pflegenden sind komplexe Beurteilungen eher der Ausnahmefall, z. B. in Fortbildungen oder Weiterbildungen, in denen Stressbewältigungs-, Konfliktlösungs- oder andere Kommunikationstrainings angeboten werden (Irving et al. 2009; Mortier et al. 2016; Walker und Mann 2016). Eine systematische, standardisierte Erfassung der Pflegequalität im Augenblick der Erbringung ist aufwendig und kostenintensiv, da sie an Verfahren der Interaktionsanalyse geknüpft ist - die auf der Verhaltensebene effektiv, aber mit einem hohen Aufwand verbunden sind und von den Pflegenden unterschiedlich nachgefragt werden (Ali u. Miller 2018; Backåberg et al. 2015).

Daher wird in der Regel auf die Erfassung der Qualität über die besser messbaren Outcomes der Pflege zurückgegriffen und die Zufriedenheit der Patientinnen und Patienten sowie ihrer An- und Zugehörigen erfasst, um über die wahrgenommene Pflegequalität Auskunft zu erhalten (Beattie et al. 2014; Fitzpatrick et al. 2014). Ob beispielsweise würdevoll gepflegt wurde, kann dadurch nur aus Einzelfallberichten eruiert werden.

\subsection{Der Ethikkodex der Pflegenden}

Dass die Pflegequalität die ethische Perspektive integriert, zeigt sich auch in den ethischen Regelwerken der Berufsgruppe. Hier ist der Ethikkodex des Council of Nursing hervorzuheben, der erstmals 1953 entworfen und in der letzten Fassung 2015 herausgegeben wurde (ANA 2015). Die Überarbeitungen erfolgten mit einem umfangreichen Reviewprozess unter Pflegenden und Pflegeexperten und in der Version der letzten Fassung sind interpretierende Statements enthalten, die eine Umsetzung der ethischen Pflichten im pflegerischen Alltag erleichtern sollen. Der Ethikkodex wird weltweit genutzt und auch in Deutschland in die Ausbildung sowie Fort-und Weiterbildung integriert.

Der oben diskutierte Würdebegriff wurde beispielsweise in einer Revision der 1960er Jahre aufgenommen und führt die neun Paragrafen an: »The nurse practices with compassion and respect for the inherent dignity, worth, and unique attributes of 
every person."(ANA 2015, S. V). Es folgen Empfehlungen für die Priorisierung des pflegerischen Handlungsauftrags (im Fokus die Pflegeempfänger/innen und deren Gesundheit), aber auch die Verantwortung zum lebenslangen Lernen und der beruflichen Interessenvertretung. Die neuen Rollen der Pflegenden in Bezug auf Evidenzbasierung und interprofessionelles Arbeiten etc. wurden ebenfalls sukzessive integriert (ANA 2015; Epstein und Turner 2015). Einige der Kriterien des Ethikkodexes könnten durchaus als pflegesensitive Kriterien herangezogen werden: Mitgefühl, Respekt, Würde, Patientenorientierung, systemischer Bezug, Sicherheit, Gesundheitsförderung, Eigenverantwortlichkeit, Entscheidungsfindung, optimale Versorgungssteuerung usw. Andere Kriterien sind auf Ebene der Patientinnen und Patienten schwieriger als Maßstab anzusetzen, sie vergesellschaften sich jedoch zum Teil mit Qualitätsindikatoren (Hasseler et al. 2016). Hierzu zählen beispielsweise die Verpflichtung zum stetigen Kompetenzausbau, zur aktiven Mitgestaltung der Professionalisierung und beruflichen Verantwortungsübernahme und interprofessionellen Arbeit (ANA 2015). Ein Ethikkodex als Regelwerk ist hilfreich, birgt jedoch auch seine Tücken: Kritisch wird es, wenn dessen Einhaltung und Reflexion durch Pflegende bei schlechten pflegerischen Rahmenbedingungen zu einem Hinterfragen des beruflichen Wirkens führen und damit zur eigenen Moralvorstellung in Kontrast stehen und eine pessimistischere Sicht auf die berufliche Selbstverwirklichung nach sich ziehen (Amiri et al. 2018; Schluter et al. 2008).

\section{- Evidenzbasierung als ethischer Handlungs- auftrag im Ethikkodex}

Der Ethikkodex verpflichtet die Pflegenden zur aktiven Mitwirkung und Mitgestaltung der Pflegeforschung und zur Umsetzung der jeweils aktuellen wissenschaftlichen Erkenntnisse zum Wohle der Patientinnen und Patienten (ANA 2015). Dies ist einerseits als Verpflichtung der Umsetzung evidenzbasierter Pflege und andererseits als Verpflichtung als Pflegeforschende formuliert (ANA 2015, S. 10). Damit wird die Evidenzbasierung nicht nur als unterstützendes Element in pflegerischen Entscheidungsfindungen, sondern als pflegerische Norm, als Wertemaßstab vorgegeben, der direkt auf die Qualität der Pflege wirkt (Riedel et al. 2017). Um eine flächendeckende evidenzbasierte Pflege zu ermöglichen, bedarf es nicht nur einer Anhebung der Zugangskriterien zum Pflegeberuf und einer regelhaften Akademisierung, sondern auch einer konsequenten Weiterbildung der bereits in der Pflege Tätigen. Die Akademisierung und kontinuierliche Registrierung oder Lizenzierung von beruflich Pflegenden kann auch als Zeichen der gesellschaftlichen Wertschätzung und des hohen Anspruchs dieses Berufs interpretiert und durch berufs- und bildungspolitische Vorgaben initiiert werden (Laiho 2010).

\subsection{Fazit}

Aus ethischer Perspektive ist eine möglichst ganzheitliche und auf die Perspektive der Patientinnen und Patienten ausgerichtete Erfassung von Pflegequalität anzustreben. Für einige der schwer zu messenden Indikatoren mit direkter Auswirkung auf die Pflegequalität (Moralvorstellungen, Haltungen, Affektkomposition, Beziehungsfähigkeit etc.) sind bereits entsprechende Messinstrumente zugänglich. Für andere Kriterien ist es neben der Frage der Messbarkeit zunächst wichtig, die pflegerische Praxis in einem ersten Schritt dafür zu sensibilisieren und die Reflexivität der Pflegenden dahingehend zu stärken. Vertrauensaufbau und Beziehungsgestaltung als pflegesensitive Outcomes zu definieren, rückt den sozialen Auftrag pflegerischer Arbeit stärker in den Fokus (Burston et al. 2014). Darüber hinaus ist eine stärkere Berücksichtigung der unsichtbaren Pflegetätigkeiten (»Missed-Care«Konzept) mit der Anerkennung der Beziehungsgestaltung verbunden, was die Wertschätzung der Pflegenden und die Motivation, Pflege weiterhin patientenorientiert auszurichten, erhöht (Sasso et al. 2017). Regelwerke wie ein Ethikkodex können den Pflegenden dabei helfen, diese Werte auch bei hoher Arbeitsdichte zu reflektieren und für sich selbst zu priorisieren. Sie können jedoch auch eine Kontrastierung des eigenen Wertesystems mit der tatsächlich leistbaren Pflege bewirken. Die Reaktionsweisen sind dichotom: Pflegende kehren ihrem Beruf den Rücken (Schürmann und Gather 2018) oder senken ihre moralischen Ansprüche (Fida et 
al. 2016). Es ist der geschichtlichen Prägung geschuldet, dass Pflegende selten aufbegehren und die berufliche Selbstverantwortung im Sinne öffentlicher Unmutsbekundungen und Streiks demonstrieren. Um eine Implosion der Pflegelandschaft in Deutschland noch abwenden zu können, wäre eine gesellschaftliche Reflexion und Diskussion dessen, was Pflege leistet und was Pflegequalität ausmacht, ebenso nötig wie eine Anerkennung, dass neue Strukturen der beruflichen und akademischen Ausund Weiterbildung und die Beschreibung neuer beruflicher Handlungsprofile benötigt werden, um den fachlichen und ethischen Ansprüchen an den Pflegeberuf gerecht zu werden.

Open Access Dieses Kapitel wird unter der Creative Commons Namensnennung 4.0 International Lizenz (http://creativecommons. org/licenses/by/4.0/deed.de) veröffentlicht, welche die Nutzung, Vervielfältigung, Bearbeitung, Verbreitung und Wiedergabe in jeglichem Medium und Format erlaubt, sofern Sie den/die ursprünglichen Autor(en) und die Quelle ordnungsgemäß nennen, einen Link zur Creative Commons Lizenz beifügen und angeben, ob Änderungen vorgenommen wurden.

Die in diesem Kapitel enthaltenen Bilder und sonstiges Drittmaterial unterliegen ebenfalls der genannten Creative Commons Lizenz, sofern sich aus der Abbildungslegende nichts anderes ergibt. Sofern das betreffende Material nicht unter der genannten Creative Commons Lizenz steht und die betreffende Handlung nicht nach gesetzlichen Vorschriften erlaubt ist, ist für die oben aufgeführten Weiterverwendungen des Materials die Einwilligung des jeweiligen Rechteinhabers einzuholen.

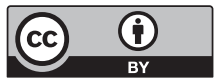

\section{Literatur}

Aiken LH, Sloane DM, Bruyneel L, van den Heede K, Griffiths $P$, Busse R, Diomidous M, Kinnunen J, Kózka M, Lesaffre E, McHugh MD, Moreno-Casbas MT, Rafferty AM, Schwendimann R, Scott PA, Tishelman C, van Achterberg T, Sermeus W (2014) Nurse staffing and education and hospital mortality in nine European countries. A retrospective observational study. The lancet 383(9931):1824-1830

Ajzen I (2011) Attitudes, personality and behavior. Open Univ. Press, Maidenhead

Ali AA, Miller ET (2018) Effectiveness of Video-Assisted Debriefing in Health Education. An Integrative Review. J Nurs Educ 57(1):14-20

Amiri E, Ebrahimi H, Vahidi M, Asghari Jafarabadi M, Namdar Areshtanab H (2018) Relationship between nurses' moral sensitivity and the quality of care. Nurs Ethics. Online first: https://doi.org/10.1177/0969733017745726. Zugegriffen: 18. Februar 2018

ANA (2015) Code of ethics for nurses with interpretive statements. American Nurses Association (ANA), Silver Spring, Maryland

Attree M (2001) Patients' and relatives' experiences and perspectives of 'Good ‘ and `Not so Good ‘ quality care. J Adv Nurs 33(4):456-466

Backåberg S, Rask M, Gummesson C, Brunt D (2015) Videobased feedback combined with reflective enquiry An interactive model for movement awareness among nursing students. Nordic Journal of Digital Literacy 10(04):246-264 ER

Batson CD (2009) These things called empathy. In: Decety J, Ickes W (Hrsg) The social neuroscience of empathy. The MIT Press

Beattie M, Lauder W, Atherton I, Murphy DJ (2014) Instruments to measure patient experience of health care quality in hospitals. A systematic review protocol. Syst Rev 3:4
Bielefeldt H (2004) Die Würde als Maßstab Philosophische Überlegungen zur Menschenrechtsbildung. In: Mahler C, Mihr A (Hrsg) Menschenrechtsbildung: Bilanz und Perspektiven. VS Verlag für Sozialwissenschaften, Wiesbaden, S 19-27

Bischoff-Wanner CM (2002) Empathie in der Pflege. Begriffsklärung und Entwicklung eines Rahmenmodells. Huber, Bern

Brunero S, Lamont S, Coates M (2010) A review of empathy education in nursing. Nurs Inq 17(1):65-74

Burston S, Chaboyer W, Gillespie B (2014) Nurse-sensitive indicators suitable to reflect nursing care quality. A review and discussion of issues. J Clin Nurs 23(13-14):1785-1795

Büscher A, Blumenberg P (2018) Nationale Expertenstandards in der Pflege - Standortbestimmung und künftige Herausforderungen. In: Hensen P, Stamer M (Hrsg) Professionsbezogene Qualitätsentwicklung im interdisziplinären Gesundheitswesen: Gestaltungsansätze, Handlungsfelder und Querschnittsbereiche. Springer Fachmedien, Wiesbaden, S 93-117

Chochinov HM (2013) Dignity in care. Time to take action. J Pain Symptom Manage 46(5):756-759

Ciompi L (2016) Die emotionalen Grundlagen des Denkens. Entwurf einer fraktalen Affektlogik. Vandenhoeck \& Ruprecht, Göttingen

Damasio AR (2015) Descartes' Irrtum. Fühlen, Denken und das menschliche Gehirn. List, München

Dinç L, Gastmans C (2013) Trust in nurse-patient relationships. A literature review. Nurs Ethics 20(5):501-516

Epstein B, Turner M (2015) The Nursing Code of Ethics. Its Value, Its History. Online J Issues Nurs 20(2):4

Fida R, Tramontano C, Paciello M, Kangasniemi M, Sili A, Bobbio A, Barbaranelli C (2016) Nurse moral disengagement. Nurs Ethics 23(5):547-564

Fitzpatrick R, Graham C, Gibbons E, King J, Flott K, Jenkinson C (2014) Development of New Models for Collection 
and Use of Patient Experience Information in the NHSPRP 070/0074. http://www.picker.org/wp-content/ uploads/2014/12/MOPE-final-report-091214.pdf. Zugegriffen: 18. Februar 2018

Foster K, McCloughen A, Delgado C, Kefalas C, Harkness E (2015) Emotional intelligence education in pre-registration nursing programmes. An integrative review. Nurse Educ Today 3:510-517

Frenk J, Chen L, Bhutta ZA, Cohen J, Crisp N, Evans T, Fineberg H, Garcia P, Ke Y, Kelley P (2010) Health professionals for a new century. Transforming education to strengthen health systems in an interdependent world. The lancet 376(9756):1923-1958.

Gillespie H, Kelly M, Duggan S, Dornan T (2017) How do patients experience caring? Scoping review. Patient Educ Couns 100(9):1622-1633

Godin G, Bélanger-Gravel A, Eccles M, Grimshaw J (2008) Healthcare professionals' intentions and behaviours. A systematic review of studies based on social cognitive theories. Implement Sci 3:36

Hasseler M, Stemmer R, Macsenaere M, Arnold J, WeidekampMaicher M (2016) Entwicklung eines wissenschaftlich basierten Qualitätsverständnisses für die Pflege- und Lebensqualität. Abschlussbericht. https://www.gkvspitzenverband.de/pflegeversicherung/qualitaet_in_ der_pflege/wissenschaftliches_qualitaetsverstaendnis/ wissenschaftliches_qualitaetsverstaendnis.jsp.

Zugegriffen: 05. Februar 2018

Heijkenskjöld KB, Ekstedt M, Lindwall L (2010) The patient's dignity from the nurse's perspective. Nurs Ethics 17(3):313-324

Irving JA, Dobkin PL, Park J (2009) Cultivating mindfulness in health care professionals. A review of empirical studies of mindfulness-based stress reduction (MBSR). Complementary therapies in clinical practice 15(2):61-66

Isfort M (2018) Fachkräftesicherung. Was tun? Heilberufe 70(1):38-39

Jacobson N (2007) Dignity and health. A review. Soc Sci Med 64(2):292-302

Jormsri P, Kunaviktikul W, Ketefian S, Chaowalit A (2005) Moral competence in nursing practice. Nurs Ethics 12(6):582-594

Kowalski CM, Rogoza R, Vernon PA, Schermer JA (2018) The Dark Triad and the self-presentation variables of socially desirable responding and self-monitoring. Personality and Individual Differences 120:234-237

Kuis EE, Hesselink G, Goossensen A (2014) Can quality from a care ethical perspective be assessed? A review. Nurs Ethics 21(7):774-793

Laiho A (2010) Academisation of nursing education in the Nordic Countries. Higher Education 60(6):641-656

Larocco SA (2010) Assisting nursing students to develop empathy using a writing assignment. Nurse Educ 35(1):10-11

Leufgen M (2018) Interkulturelle und interreligiöse Kompetenzen als Qualitätsmerkmal professioneller Handlungspraxis. In: Hensen P, Stamer M (Hrsg). Professionsbezogene Qualitätsentwicklung im interdisziplinären Gesundheitswesen: Gestaltungsansätze, Handlungs- felder und Querschnittsbereiche. Springer Fachmedien, Wiesbaden, S 311-340

Mortier AV, Vlerick P, Clays E (2016) Authentic leadership and thriving among nurses: The mediating role of empathy. J Nurs Manag 24(3):357-365

Riedel A, Behrens J, Giese C, Geiselhart M, Fuchs G, Kohlen H, Pasch W, Rabe M, Schütze L (2017) Zentrale Aspekte der Ethikkompetenz in der Pflege. Ethik Med 29(2):161-165

Sasso L, Bagnasco A, Aleo G, Catania G, Dasso N, Zanini MP, Watson R (2017) Incorporating nursing complexity in reimbursement coding systems. The potential impact on missed care. BMJ Qual Saf 26(11):929-932

Schluter J, Winch S, Holzhauser K, Henderson A (2008) Nurses' moral sensitivity and hospital ethical climate. A literature review. Nurs Ethics 15(3):304-321

Schreyögg J, Milstein R (2016) Expertise zur Ermittlung des Zusammenhangs zwischen Pflegeverhältniszahlen und pflegesensitiven Ergebnisparametern in Deutschland. https://www.bundesgesundheitsministerium.de/ fileadmin/Dateien/5_Publikationen/Pflege/ Berichte/Gutachten_Schreyoegg_Pflegesensitive_ Fachabteilungen.pdf. Zugegriffen: 18. Februar 2018

Schürmann L, Gather C (2018) Pflegearbeit im Wandel. In: Bührmann AD, Fachinger U, Welskop-Deffaa EM (Hrsg). Hybride Erwerbsformen: Digitalisierung, Diversität und sozialpolitische Gestaltungsoptionen. Springer Fachmedien, Wiesbaden, S 157-187

Schwarz R (2009) Supervision und professionelles Handeln Pflegender. Wiesbaden: VS Verlag für Sozialwissenschaften /GWV Fachverlage GmbH, Wiesbaden

Sinclair S, Beamer K, Hack T, McClement S, Boucha SR, Chochinov H, Hagen N (2017) Sympathy, empathy, and compassion. A grounded theory study of palliative care patients' understandings, experiences, and preferences. Palliative Medicine 31(5):437-447

Staudhammer M (2018) Konflikt- und Spannungsfelder in der Pflege. In: Prävention von Machtmissbrauch und Gewalt in der Pflege. Springer, Berlin Heidelberg, S 95-115

Stievano A, Rocco G, Sabatino L, Alvaro R (2013) Dignity in Professional Nursing. Guaranteeing Better Patient Care. Journal of Radiology Nursing 32(3):120-123.

Walker M, Mann RA (2016) Exploration of mindfulness in relation to compassion, empathy and reflection within nursing education. Nurse Educ Today 40:188-190.

WHO (1994) A Declaration on the promotion of patients' rights in Europe: European Consultation on the Rights of Patients. http://www.who.int/genomics/public/eu_ declaration1994.pdf. Zugegriffen: 06. Februar 2018

Wilson R, Godfrey CM, Sears K, Medves J, Ross-White A, Lambert $\mathrm{N}$ (2015) Exploring conceptual and theoretical frameworks for nurse practitioner education. A scoping review protocol. JBI Database System Rev Implement Rep 13(10):146-155

Yu J, Kirk M (2009) Evaluation of empathy measurement tools in nursing. systematic review. J Adv Nurs 65(9):1790-1806

Zhang Z-x, Luk W, Arthur D, Wong T (2001) Nursing competencies. Personal characteristics contributing to effective nursing performance. J Adv Nurs 33(4):467-474 